Research Paper

\title{
Identification of polymorphisms in 12q24.1, ACAD10, and BRAP as novel genetic determinants of blood pressure in Japanese by exome-wide association studies
}

\author{
Yoshiji Yamada ${ }^{1,2}$, Jun Sakuma ${ }^{2,3,4}$, Ichiro Takeuchi ${ }^{2,4,5}$, Yoshiki Yasukochi ${ }^{1,2}$, \\ Kimihiko Kato ${ }^{1,6}$, Mitsutoshi Oguri,1,7, Tetsuo Fujimaki, Hideki Horibe ${ }^{9}$, Masaaki \\ Muramatsu ${ }^{10}$, Motoji Sawabe ${ }^{11}$, Yoshinori Fujiwara ${ }^{12}$, Yu Taniguchi' ${ }^{12}$ Shuichi \\ Obuchi ${ }^{13}$, Hisashi Kawai ${ }^{13}$, Shoji Shinkai ${ }^{14}$, Seijiro Mori ${ }^{15}$, Tomio Arai ${ }^{16}$ and Masashi \\ Tanaka ${ }^{17}$ \\ ${ }^{1}$ Department of Human Functional Genomics, Advanced Science Research Promotion Center, Mie University, Tsu, Japan \\ ${ }^{2}$ CREST, Japan Science and Technology Agency, Kawaguchi, Japan \\ ${ }^{3}$ Computer Science Department, College of Information Science, University of Tsukuba, Tsukuba, Japan \\ ${ }^{4}$ RIKEN Center for Advanced Intelligence Project, Tokyo, Japan \\ ${ }^{5}$ Department of Computer Science, Nagoya Institute of Technology, Nagoya, Japan \\ ${ }^{6}$ Department of Internal Medicine, Meitoh Hospital, Nagoya, Japan \\ ${ }^{7}$ Department of Cardiology, Kasugai Municipal Hospital, Kasugai, Japan \\ ${ }^{8}$ Department of Cardiovascular Medicine, Inabe General Hospital, Inabe, Japan \\ ${ }^{9}$ Department of Cardiovascular Medicine, Gifu Prefectural Tajimi Hospital, Tajimi, Japan \\ ${ }^{10}$ Department of Molecular Epidemiology, Medical Research Institute, Tokyo Medical and Dental University, Tokyo, Japan \\ ${ }^{11}$ Section of Molecular Pathology, Graduate School of Health Care Sciences, Tokyo Medical and Dental University, Tokyo, Japan \\ ${ }^{12}$ Research Team for Social Participation and Community Health, Tokyo Metropolitan Institute of Gerontology, Tokyo, Japan \\ ${ }^{13}$ Research Team for Promoting Support System for Home Care, Tokyo Metropolitan Institute of Gerontology, Tokyo, Japan \\ ${ }^{14}$ Research Team for Social Participation and Health Promotion, Tokyo Metropolitan Institute of Gerontology, Tokyo, Japan \\ ${ }^{15}$ Center for Promotion of Clinical Investigation, Tokyo Metropolitan Geriatric Hospital, Tokyo, Japan \\ ${ }^{16}$ Department of Pathology, Tokyo Metropolitan Geriatric Hospital, Tokyo, Japan \\ ${ }^{17}$ Department of Clinical Laboratory, Tokyo Metropolitan Geriatric Hospital, Tokyo, Japan \\ Correspondence to: Yoshiji Yamada, email: yamada@gene.mie-u.ac.jp \\ Keywords: hypertension, blood pressure, genetics, exome-wide association study, polymorphism \\ Received: March 19, $2017 \quad$ Accepted: April 05, $2017 \quad$ Published: April 27, 2017 \\ Copyright: Yamada et al. This is an open-access article distributed under the terms of the Creative Commons Attribution License \\ 3.0 (CC BY 3.0), which permits unrestricted use, distribution, and reproduction in any medium, provided the original author and \\ source are credited.
}

\section{ABSTRACT}

We performed exome-wide association studies to identify genetic variants that influence systolic or diastolic blood pressure or confer susceptibility to hypertension in Japanese. The exome-wide association studies were performed with the use of Illumina HumanExome-12 DNA Analysis BeadChip or Infinium Exome-24 BeadChip arrays and with 14,678 subjects, including 8215 individuals with hypertension and 6463 controls. The relation of genotypes of 41,843 single nucleotide polymorphisms to systolic or diastolic blood pressure was examined by linear regression analysis. After Bonferroni's correction, 44 and eight polymorphisms were significantly $(P<$ $1.19 \times 10^{-6}$ ) associated with systolic or diastolic blood pressure, respectively, with six polymorphisms (rs12229654, rs671, rs11066015, rs2074356, rs3782886, rs11066280) being associated with both systolic and diastolic blood pressure. Examination of the relation of allele frequencies to hypertension with Fisher's exact test revealed that 
100 of the 41,843 single nucleotide polymorphisms were significantly $\left(P<1.19 \times 10^{-}\right.$ $\left.{ }^{6}\right)$ associated with hypertension. Subsequent multivariable logistic regression analysis with adjustment for age and sex showed that five polymorphisms (rs150854849, rs202069030, rs139012426, rs12229654, rs76974938) were significantly $(P<1.25$ $\times 10^{-4}$ ) associated with hypertension. The polymorphism rs12229654 was thus associated with both systolic and diastolic blood pressure and with hypertension. Six polymorphisms (rs12229654 at 12q24.1, rs671 of ALDH2, rs11066015 of ACAD10, rs2074356 and rs11066280 of HECTD4, and rs3782886 of BRAP) were found to be associated with both systolic and diastolic blood pressure, with those at 12q24.1 or in ACAD10 or BRAP being novel determinants of blood pressure in Japanese.

\section{INTRODUCTION}

Hypertension is a major risk factor for coronary artery disease and stroke [1], with a heritability estimated to be $\sim 30 \%$ [2]. Genome-wide association studies (GWASs) have implicated various loci and genes in predisposition to hypertension in populations of European [3-6] or African [7] ancestry as well as in Chinese [8] and Japanese [9] individuals. A recent trans-ancestry GWAS in individuals of European and East or South Asian ancestry identified 12 loci associated with blood pressure (BP) [10]. Most of the genetic variants identified in these studies have a minor allele frequency (MAF) of $\geq 5 \%$ and a small individual effect size. Given that these common variants explain only a fraction of the heritability of hypertension, low-frequency $(0.5 \% \leq \mathrm{MAF}<5 \%)$ or rare (MAF $<0.5 \%$ ) variants with larger effect sizes likely contribute to the genetic architecture of this condition [11].

We have now performed exome-wide association studies (EWASs) with the use of exome array-based genotyping methods to identify single nucleotide polymorphisms (SNPs) - in particular, low-frequency or rare coding variants with moderate to large effect sizesthat confer susceptibility to elevated BP or hypertension in Japanese. We applied Illumina human exome arrays that provide coverage of functional SNPs in entire exons including low-frequency and rare variants.

\section{RESULTS}

\section{EWASs for systolic and diastolic BP}

We examined the relation of genotypes for 41,843 SNPs that passed quality control to systolic or diastolic BP by linear regression analysis. Manhattan plots of the EWASs for systolic and diastolic BP are shown in Supplementary Figure 1. After Bonferroni's correction, 44 or eight SNPs were significantly $\left(P<1.19 \times 10^{-6}\right)$ associated with systolic (Table 1) or diastolic (Table 2) BP, respectively. Among these SNPs, six polymorphisms (rs12229654 at 12q24.1, rs671 of $A L D H 2$, rs11066015 of $A C A D 10$, rs2074356 and rs11066280 of HECTD4, rs3782886 of $B R A P$ ) were significantly associated with both systolic and diastolic BP.

\section{EWAS for hypertension}

We next performed an EWAS for hypertension. The characteristics of the subjects enrolled in the study are shown in Table 3. Age, the frequency of men, body mass index, and the prevalence of diabetes mellitus, dyslipidemia, chronic kidney disease, and hyperuricemia were greater, whereas the prevalence of smoking was lower, in subjects with hypertension than in controls. We examined the relation of allele frequencies of $41,843 \mathrm{SNPs}$ to hypertension with Fisher's exact test. A Manhattan plot of the EWAS for hypertension is shown in Supplementary Figure 1. After Bonferroni's correction, 100 SNPs were significantly $\left(P<1.19 \times 10^{-6}\right)$ associated with hypertension (Supplementary Table 1). The genotype distributions of these SNPs were in Hardy-Weinberg equilibrium ( $P$ $\geq 0.001$ ) among both subjects with hypertension and controls (Supplementary Table 2).

\section{Multivariable logistic regression analysis of the} relation of SNPs to hypertension

The relation of the 100 SNPs identified in the EWAS for hypertension to this condition was further examined by multivariable logistic regression analysis with adjustment for age and sex (Supplementary Table 3). Nine SNPs were related $(P<0.01$ in any one genetic model) to hypertension (Table 4), and five of these SNPs (rs150854849 of DCLRE1C, rs202069030 of DUS2, rs139012426 of LOC100505549, rs12229654 at 12q24.1, rs76974938 of C21orf59) were significantly $\left(P<1.25 \times 10^{-4}\right)$ associated with this condition. The minor $T$ allele of rs 150854849 was a risk factor for hypertension, whereas the minor $C$ (rs202069030), C (rs139012426), G (rs12229654), and $T$ (rs76974938) alleles of the other four SNPs were protective against this condition. The rs 12229654 SNP at 12q24.1 was significantly associated with both systolic and diastolic BP as well as with hypertension.

\section{Relation of SNPs identified in the present study to systolic or diastolic BP}

We next examined the relation of genotypes for the 50 SNPs isolated in the present study to systolic or diastolic BP by one-way analysis of variance (ANOVA) (Table 5). The SNP rs12229654, which was identified 
Table 1: The 44 single nucleotide polymorphisms (SNPs) significantly $\left(P<1.19 \times 10^{-6}\right)$ associated with systolic blood pressure by the exome-wide association study

\begin{tabular}{|c|c|c|c|c|c|}
\hline Gene & dbSNP & $\begin{array}{l}\text { Nucleotide } \\
\text { (amino acid) } \\
\text { substitution }^{\mathrm{a}}\end{array}$ & $\begin{array}{c}\text { Chromosome: } \\
\text { position }\end{array}$ & MAF (\%) & $P$ (genotype) \\
\hline MUC17 & rs78010183 & A/T (T1305S) & 7: 101035329 & 1.8 & $3.86 \times 10^{-32}$ \\
\hline OR4F6 & rs141569282 & G/A (A117T) & 15: 101806068 & 1.7 & $1.10 \times 10^{-27}$ \\
\hline COL6A5 & rs200982668 & G/A (E2501K) & 3: 130470894 & 1.3 & $2.45 \times 10^{-22}$ \\
\hline$M A R C H 1$ & rs61734696 & G/T (Q137K) & 4: 164197303 & 1.2 & $3.48 \times 10^{-22}$ \\
\hline PLCB2 & rs200787930 & C/T (E1095K) & 15: 40289298 & 1.2 & $6.54 \times 10^{-22}$ \\
\hline$M O B 3 C$ & rs139537100 & C/T (R24Q) & 1: 46615006 & 1.2 & $2.54 \times 10^{-21}$ \\
\hline$V P S 33 B$ & rs199921354 & C/T (R80Q) & 15: 91013841 & 1.2 & $4.06 \times 10^{-21}$ \\
\hline CXCL8 & rs188378669 & G/T (E31*) & 4: 73741568 & 1.2 & $1.40 \times 10^{-20}$ \\
\hline COL6A3 & rs146092501 & C/T (E1386K) & 2: 237371861 & 1.2 & $2.45 \times 10^{-20}$ \\
\hline ZNF77 & rs146879198 & G/A (R340*) & 19: 2934109 & 1.2 & $2.70 \times 10^{-20}$ \\
\hline TMOD4 & rs115287176 & G/A (R277W) & 1: 151170961 & 1.2 & $5.08 \times 10^{-20}$ \\
\hline$A D G R L 3$ & rs192210727 & G/T (R580I) & 4: 61909615 & 1.3 & $1.20 \times 10^{-19}$ \\
\hline PRAMEF 12 & rs199576535 & G/A (V341I) & 1: 12777168 & 1.0 & $5.70 \times 10^{-16}$ \\
\hline PTCH2 & rs 147284320 & C/T (V503I) & 1: 44828589 & 2.0 & $4.36 \times 10^{-14}$ \\
\hline \multirow[t]{2}{*}{$I G S F 9 B$} & rs201459911 & G/A (A1115V) & 11: 133920381 & 0.7 & $6.77 \times 10^{-14}$ \\
\hline & rs12229654 & $\mathrm{T} / \mathrm{G}$ & 12: 110976657 & 22.5 & $2.04 \times 10^{-11}$ \\
\hline$A L D H 2$ & rs671 & G/A (E504K) & 12: 111803962 & 27.6 & $3.97 \times 10^{-11}$ \\
\hline$A C A D 10$ & rs 11066015 & $\mathrm{G} / \mathrm{A}$ & 12: 111730205 & 27.5 & $8.38 \times 10^{-11}$ \\
\hline HECTD4 & rs2074356 & $\mathrm{C} / \mathrm{T}$ & 12: 112207597 & 25.4 & $4.48 \times 10^{-10}$ \\
\hline$B R A P$ & rs3782886 & $\mathrm{A} / \mathrm{G}$ & 12: 111672685 & 29.3 & $2.82 \times 10^{-9}$ \\
\hline HECTD4 & rs11066280 & $\mathrm{T} / \mathrm{A}$ & 12: 112379979 & 29.0 & $3.38 \times 10^{-9}$ \\
\hline \multirow[t]{2}{*}{$R N F 213$} & rs199976159 & G/A (G222S) & $17: 80288217$ & 0.3 & $3.98 \times 10^{-9}$ \\
\hline & rs2523638 & $\mathrm{G} / \mathrm{A}$ & $6: 31376496$ & 43.1 & $1.12 \times 10^{-8}$ \\
\hline \multirow[t]{2}{*}{$A S 3 M T$} & rs11191454 & $\mathrm{A} / \mathrm{G}$ & 10: 102900247 & 26.8 & $2.39 \times 10^{-8}$ \\
\hline & rs12182351 & $\mathrm{T} / \mathrm{C}$ & $6: 32233930$ & 29.8 & $2.42 \times 10^{-8}$ \\
\hline CNNM2 & rs12413409 & $\mathrm{G} / \mathrm{A}$ & 10: 102959339 & 26.4 & $3.24 \times 10^{-8}$ \\
\hline \multirow[t]{2}{*}{ NPFFR2 } & rs144936999 & G/C (A332P) & 4: 72147237 & 0.2 & $4.16 \times 10^{-8}$ \\
\hline & rs404890 & $\mathrm{G} / \mathrm{T}$ & $6: 32231090$ & 30.5 & $4.94 \times 10^{-8}$ \\
\hline CCHCR1 & rs 130075 & C/T (R102Q) & $6: 31154725$ & 13.2 & $5.07 \times 10^{-8}$ \\
\hline NT5C2 & rs11191580 & $\mathrm{T} / \mathrm{C}$ & 10: 103146454 & 26.3 & $6.68 \times 10^{-8}$ \\
\hline$H L A-B$ & rs 1058026 & $\mathrm{~T} / \mathrm{G}$ & $6: 31353908$ & 33.4 & $9.03 \times 10^{-8}$ \\
\hline CNNM2 & rs11191548 & $\mathrm{T} / \mathrm{C}$ & 10: 103086421 & 26.3 & $9.31 \times 10^{-8}$ \\
\hline C6orf15 & rs2270191 & C/T (V5M) & $6: 31112543$ & 12.4 & $1.79 \times 10^{-7}$ \\
\hline$C D S N$ & rs117951780 & $\mathrm{C} / \mathrm{T}(\mathrm{S} 453 \mathrm{~N})$ & $6: 31116257$ & 12.3 & $2.45 \times 10^{-7}$ \\
\hline C6orf15 & rs2270190 & $\mathrm{T} / \mathrm{C}$ & 6: 31112809 & 12.9 & $\begin{array}{l}2.96 \times 10^{-7} \\
(\text { Continued }\end{array}$ \\
\hline
\end{tabular}




\begin{tabular}{lccccc}
\hline Gene & dbSNP & $\begin{array}{c}\text { Nucleotide } \\
\text { (amino acid) } \\
\text { substitution }^{\mathbf{a}}\end{array}$ & $\begin{array}{c}\text { Chromosome: } \\
\text { position }\end{array}$ & MAF (\%) & P (genotype) \\
\hline CYP17A1 & rs17115100 & $\mathrm{G} / \mathrm{T}$ & $10: 102831636$ & 33.5 & $3.44 \times 10^{-7}$ \\
PSORS1C1 & $\mathrm{rs} 72655343$ & $\mathrm{C} / \mathrm{A}$ & $11: 1104741$ & 0.1 & $4.24 \times 10^{-7}$ \\
CCHCR1 & $\mathrm{r} 1063646$ & $\mathrm{C} / \mathrm{T}$ (P133L) & $6: 31139871$ & 12.9 & $5.26 \times 10^{-7}$ \\
CYP17A1 & $\mathrm{rs9263739}$ & $\mathrm{C} / \mathrm{T}$ & $6: 31143579$ & 12.9 & $5.26 \times 10^{-7}$ \\
CAT & $\mathrm{rs} 1004467$ & $\mathrm{~T} / \mathrm{C}$ & $10: 102834750$ & 32.4 & $5.63 \times 10^{-7}$ \\
PSORS1C2 & $\mathrm{rs} 139421991$ & $\mathrm{G} / \mathrm{A}(\mathrm{R} 320 \mathrm{Q})$ & $11: 34456720$ & 0.3 & $6.74 \times 10^{-7}$ \\
RNF39 & $\mathrm{rs} 7757012$ & $\mathrm{~T} / \mathrm{C}$ & $6: 31140785$ & 13.0 & $8.23 \times 10^{-7}$ \\
CCDC63 & $\mathrm{rs} 142979264$ & $\mathrm{C} / \mathrm{T}$ & $6: 30075223$ & 11.8 & $9.58 \times 10^{-7}$ \\
\hline
\end{tabular}

The relation of genotypes of SNPs to systolic blood pressure was analyzed with a linear regression model. ${ }^{a}$ Major allele/ minor allele. MAF, minor allele frequency.

Table 2: The eight single nucleotide polymorphisms (SNPs) significantly $\left(P<1.19 \times 10^{-6}\right)$ associated with diastolic blood pressure by the exome-wide association study

\begin{tabular}{lccccc}
\hline Gene & dbSNP & $\begin{array}{c}\text { Nucleotide } \\
\text { (amino acid) }_{\text {substitution }^{\mathbf{a}}}\end{array}$ & $\begin{array}{c}\text { Chromosome: } \\
\text { position }\end{array}$ & MAF (\%) & $\boldsymbol{P}$ (genotype) \\
\hline ALDH2 & $\mathrm{rs} 671$ & $\mathrm{G} / \mathrm{A}(\mathrm{E} 504 \mathrm{~K})$ & $12: 111803962$ & 27.6 & $2.18 \times 10^{-15}$ \\
ACAD10 & $\mathrm{rs} 11066015$ & $\mathrm{G} / \mathrm{A}$ & $12: 111730205$ & 27.5 & $4.08 \times 10^{-15}$ \\
BRAP & $\mathrm{rs} 3782886$ & $\mathrm{~A} / \mathrm{G}$ & $12: 111672685$ & 29.3 & $2.46 \times 10^{-14}$ \\
HECTD4 & $\mathrm{rs} 11066280$ & $\mathrm{~T} / \mathrm{A}$ & $12: 112379979$ & 29.0 & $8.38 \times 10^{-14}$ \\
HECTD4 & $\mathrm{rs} 2074356$ & $\mathrm{C} / \mathrm{T}$ & $12: 112207597$ & 25.4 & $2.41 \times 10^{-13}$ \\
& $\mathrm{rs} 12229654$ & $\mathrm{~T} / \mathrm{G}$ & $12: 110976657$ & 22.5 & $2.16 \times 10^{-12}$ \\
ATXN2 & $\mathrm{rs} 7969300$ & $\mathrm{~T} / \mathrm{C}(\mathrm{N} 248 \mathrm{~S})$ & $12: 111555908$ & 38.8 & $2.62 \times 10^{-8}$ \\
NAA25 & $\mathrm{rs} 12231744$ & $\mathrm{C} / \mathrm{T}(\mathrm{R} 876 \mathrm{~K})$ & $12: 112039251$ & 35.1 & $6.76 \times 10^{-7}$ \\
\hline
\end{tabular}

The relation of genotypes of SNPs to diastolic blood pressure was analyzed with a linear regression model. ${ }^{a}$ Major allele/ minor allele. MAF, minor allele frequency.

in the EWASs for systolic and diastolic BP and hypertension, was significantly $(P<0.0005)$ associated with both systolic and diastolic BP. The SNPs rs671, rs11066015, rs2074356, rs3782886, and rs11066280 identified in the EWASs for systolic and diastolic BP were also significantly associated with both systolic and diastolic BP. In addition, rs 141569282 of $O R 4 F 6$, rs 2523638 at $6 \mathrm{p} 21.3$, and rs 10849915 of CCDC63 identified in the EWAS of systolic BP were significantly associated with both systolic and diastolic BP. The SNPs rs150854849, rs202069030, rs139012426, and rs76974938 identified in the EWAS for hypertension were not significantly associated with either systolic or diastolic BP, probably because of the effect of antihypertensive treatment.

\section{Linkage disequilibrium and haplotype analysis}

Given that the six SNPs (rs12229654, rs671, rs11066015, rs2074356, rs3782886, rs11066280) found to be associated with both systolic and diastolic BP are all located at chromosomal region 12q24.12-q24.13, we examined linkage disequilibrium among these polymorphisms as well as the relation of their haplotypes to hypertension. The six SNPs were all in strong linkage disequilibrium (Supplementary Table 4). Haplotype analysis revealed that the haplotypes $T$ (rs12229654)-G (rs671)-G (rs11066015)-C (rs2074356)-A (rs3782886)-T (rs11066280) and G (rs12229654)-A (rs671)-A (rs11066015)-T (rs2074356)-G (rs3782886)-A $\left(\right.$ rs11066280) were significantly $\left(P<9.62 \times 10^{-4}\right)$ 
Table 3: Characteristics of the 14,678 study subjects

\begin{tabular}{lccc}
\hline Characteristic & Hypertension & Controls & $\boldsymbol{P}$ \\
\hline No. of subjects & 8215 & 6463 & $<0.0001$ \\
Age (years) & $67.2 \pm 12.1$ & $55.8 \pm 14.0$ & $<0.0001$ \\
Sex (male/female, \%) & $59.6 / 40.4$ & $51.4 / 48.6$ & $<0.0001$ \\
Body mass index (kg/m ${ }^{2}$ ) & $23.9 \pm 3.6$ & $22.6 \pm 3.2$ & 0.0021 \\
Current or former smoker & 35.3 & 37.9 & $<0.0001$ \\
(\%) & 35.3 & 12.1 & $<0.0001$ \\
Diabetes mellitus (\%) & 69.6 & 51.7 & $<0.0001$ \\
Dyslipidemia (\%) & 32.4 & 14.0 & $<0.0001$ \\
Chronic kidney disease (\%) & 23.6 & 11.1 & $<0.0001$ \\
Hyperuricemia (\%) & $144 \pm 24$ & $116 \pm 12$ & $<0.0001$ \\
Systolic blood pressure & & \\
(mmHg) & $81 \pm 14$ & $70 \pm 10$ & \\
Diastolic blood pressure & & \\
(mmHg) & & & \\
\hline
\end{tabular}

Quantitative data are means \pm SD and were compared between subjects with hypertension and controls with the unpaired Student's $t$ test. Categorical data were compared between the two groups with Fisher's exact test. Based on Bonferroni's correction, a $P$ value of $<0.0050(0.05 / 10)$ was considered statistically significant.

Table 4: Relation of SNPs to hypertension as determined by multivariable logistic regression analysis

\begin{tabular}{|c|c|c|c|c|c|c|c|c|c|}
\hline \multirow[b]{2}{*}{ SNP } & & \multicolumn{2}{|c|}{ Dominant } & \multicolumn{2}{|r|}{ Recessive } & \multicolumn{2}{|c|}{ Additive 1} & \multicolumn{2}{|c|}{ Additive 2} \\
\hline & & $P$ & OR $(95 \%$ CI $)$ & $P$ & OR $(95 \%$ CI) & $P$ & OR $(95 \%$ CI $)$ & $P$ & $\begin{array}{c}\text { OR } \\
(95 \% \mathrm{CI})\end{array}$ \\
\hline rs150854849 & C/T (R179Q) & $1.90 \times 10^{-16}$ & $6.53(3.79-12.41)$ & ND & & $1.90 \times 10^{-16}$ & $6.53(3.79-12.41)$ & ND & \\
\hline rs 2867125 & $\mathrm{G} / \mathrm{A}$ & 0.0058 & $0.88(0.80-0.96)$ & 0.4777 & & 0.0076 & $0.88(0.80-0.97)$ & 0.4056 & \\
\hline rs 202069030 & G/C (R51S) & $1.01 \times 10^{-15}$ & $0.15(0.08-0.25)$ & ND & & $1.01 \times 10^{-15}$ & $0.15(0.08-0.25)$ & ND & \\
\hline rs 139012426 & $\mathrm{G} / \mathrm{C}(\mathrm{S} 1242 \mathrm{~T})$ & $7.12 \times 10^{-11}$ & $0.24(0.15-0.38)$ & ND & & $7.12 \times 10^{-11}$ & $0.24(0.15-0.38)$ & ND & \\
\hline rs 75432131 & G/A (H606Y) & 0.0046 & $1.36(1.10-1.69)$ & 0.2511 & & 0.0065 & $1.35(1.09-1.67)$ & 0.2473 & \\
\hline rs12229654 & $\mathrm{T} / \mathrm{G}$ & $1.13 \times 10^{-6}$ & $0.83(0.77-0.90)$ & 0.0132 & $0.82(0.70-0.96)$ & $1.44 \times 10^{-5}$ & $0.84(0.78-0.91)$ & 0.0014 & $\begin{array}{c}0.77 \\
(0.65-0.90)\end{array}$ \\
\hline rs76974938 & $\mathrm{C} / \mathrm{T}(\mathrm{D} 67 \mathrm{~N})$ & $5.14 \times 10^{-5}$ & $0.69(0.57-0.82)$ & ND & & $5.14 \times 10^{-5}$ & $0.69(0.57-0.82)$ & ND & \\
\hline rs201633733 & $\mathrm{A} / \mathrm{G}$ & 0.0025 & $2.80(1.43-5.73)$ & ND & & 0.0025 & $2.80(1.43-5.73)$ & ND & \\
\hline rs 12352313 & A/G (N901S) & 0.0086 & $0.89(0.81-0.97)$ & 0.0518 & & 0.0240 & $0.90(0.82-0.99)$ & 0.0394 & $\begin{array}{c}0.70 \\
(0.51-0.98)\end{array}$ \\
\hline
\end{tabular}

Multivariable logistic regression analysis was performed with adjustment for age and sex. Based on Bonferroni's correction, $P$ values of $<1.25 \times$ $10^{-4}(0.05 / 400)$ were considered statistically significant and are shown in bold. OR, odds ratio; CI, confidence interval; ND, not determined.

associated with hypertension, with the former haplotype representing a risk factor for and the latter being protective against this condition (Supplementary Table 5).

\section{Relation of chromosomal loci, genes, and SNPs identified in the present study to phenotypes examined in previous GWASs}

Finally, we examined the relation of the 50 SNPs of 44 genes or chromosomal loci identified in the present study to phenotypes previously probed in GWASs that are available in a public database [GWAS Catalog (National Human Genome Research Institute and European Bioinformatics Institute), http://www. ebi.ac.uk/gwas] (Supplementary Table 6). ALDH2 [10], HECTD4 [10], AS3MT [12], CNNM2 [6, 10, 13, 14], NT5C2 [10], and CYP17A1 [4, 10] were previously identified as loci associated with BP in previous GWASs. 
Table 5: Relation of SNPs to systolic or diastolic blood pressure (BP)

\begin{tabular}{|c|c|c|c|c|c|c|c|c|c|}
\hline \multicolumn{2}{|l|}{ SNP } & \multicolumn{3}{|c|}{ Systolic BP (mmHg) } & $\boldsymbol{P}$ & \multicolumn{3}{|c|}{ Diastolic BP (mmHg) } & \multirow[t]{2}{*}{$P$} \\
\hline \multicolumn{9}{|c|}{ Associated with systolic and diastolic BP and hypertension } & \\
\hline \multirow[t]{2}{*}{ rs12229654 } & $\mathrm{T} / \mathrm{G}$ & $T T$ & $T G$ & $G G$ & & $T T$ & $T G$ & $G G$ & \\
\hline & & $132 \pm 24$ & $130 \pm 23$ & $128 \pm 24$ & $1.16 \times 10^{-10}$ & $77 \pm 14$ & $75 \pm 13$ & $75 \pm 13$ & $6.50 \times 10^{-12}$ \\
\hline \multicolumn{10}{|c|}{ Associated with systolic and diastolic BP } \\
\hline \multirow[t]{2}{*}{ rs671 } & $\mathrm{G} / \mathrm{A}(\mathrm{E} 504 \mathrm{~K})$ & $G G$ & $G A$ & $A A$ & & $G G$ & $G A$ & $A A$ & \\
\hline & & $132 \pm 24$ & $130 \pm 23$ & $129 \pm 24$ & $2.32 \times 10^{-11}$ & $77 \pm 14$ & $75 \pm 13$ & $75 \pm 13$ & $5.02 \times 10^{-15}$ \\
\hline \multirow[t]{2}{*}{ rs11066015 } & $\mathrm{G} / \mathrm{A}$ & $G G$ & $G A$ & $A A$ & & $G G$ & $G A$ & $A A$ & \\
\hline & & $132 \pm 24$ & $130 \pm 23$ & $129 \pm 24$ & $3.87 \times 10^{-11}$ & $77 \pm 14$ & $75 \pm 13$ & $75 \pm 13$ & $7.37 \times 10^{-15}$ \\
\hline \multirow[t]{2}{*}{ rs2074356 } & $\mathrm{C} / \mathrm{T}$ & $C C$ & $C T$ & $T T$ & & $C C$ & $C T$ & $T T$ & \\
\hline & & $132 \pm 24$ & $130 \pm 23$ & $129 \pm 24$ & $9.57 \times 10^{-10}$ & $77 \pm 14$ & $75 \pm 13$ & $74 \pm 13$ & $1.40 \times 10^{-12}$ \\
\hline \multirow[t]{2}{*}{ rs3782886 } & $\mathrm{A} / \mathrm{G}$ & $A A$ & $A G$ & $G G$ & & $A A$ & $A G$ & $G G$ & \\
\hline & & $132 \pm 24$ & $130 \pm 23$ & $129 \pm 24$ & $2.38 \times 10^{-9}$ & $77 \pm 14$ & $75 \pm 13$ & $75 \pm 13$ & $4.40 \times 10^{-14}$ \\
\hline \multirow[t]{2}{*}{ rs11066280 } & $\mathrm{T} / \mathrm{A}$ & $T T$ & $T A$ & $A A$ & & $T T$ & $T A$ & $A A$ & \\
\hline & & $132 \pm 24$ & $130 \pm 23$ & $129 \pm 24$ & $4.71 \times 10^{-9}$ & $77 \pm 14$ & $75 \pm 13$ & $75 \pm 13$ & $9.81 \times 10^{-14}$ \\
\hline \multicolumn{10}{|c|}{ Associated with systolic BP } \\
\hline \multirow[t]{2}{*}{ rs78010183 } & $\mathrm{A} / \mathrm{T}(\mathrm{T} 1305 \mathrm{~S})$ & $A A$ & $A T$ & & & $A A$ & $A T$ & & \\
\hline & & $132 \pm 24$ & $120 \pm 16$ & & $<1.0 \times 10^{-23}$ & $76 \pm 14$ & $74 \pm 12$ & & 0.0011 \\
\hline \multirow[t]{2}{*}{ rs141569282 } & G/A (A117T) & $G G$ & $G A$ & & & $G G$ & $G A$ & & \\
\hline & & $134 \pm 25$ & $121 \pm 17$ & & $<1.0 \times 10^{-23}$ & $77 \pm 14$ & $74 \pm 12$ & & 0.0002 \\
\hline \multirow[t]{2}{*}{ rs200982668 } & G/A (E2501K) & $G G$ & $G A$ & & & $G G$ & $G A$ & & \\
\hline & & $131 \pm 24$ & $120 \pm 16$ & & $2.50 \times 10^{-22}$ & $76 \pm 14$ & $75 \pm 12$ & & 0.0473 \\
\hline \multirow[t]{2}{*}{ rs61734696 } & G/T (Q137K) & $G G$ & $G T$ & & & $G G$ & $G T$ & & \\
\hline & & $131 \pm 24$ & $119 \pm 16$ & & $3.50 \times 10^{-22}$ & $76 \pm 14$ & $75 \pm 12$ & & 0.0303 \\
\hline \multirow[t]{2}{*}{ rs 200787930} & $\mathrm{C} / \mathrm{T}(\mathrm{E} 1095 \mathrm{~K})$ & $C C$ & $C T$ & & & $C C$ & $C T$ & & \\
\hline & & $131 \pm 24$ & $119 \pm 16$ & & $6.50 \times 10^{-22}$ & $76 \pm 14$ & $75 \pm 12$ & & 0.0640 \\
\hline \multirow[t]{2}{*}{ rs139537100 } & $\mathrm{C} / \mathrm{T}$ (R24Q) & $C C$ & $C T$ & & & $C C$ & $C T$ & & \\
\hline & & $131 \pm 24$ & $120 \pm 16$ & & $2.54 \times 10^{-21}$ & $76 \pm 14$ & $75 \pm 12$ & & 0.0449 \\
\hline \multirow[t]{2}{*}{ rs199921354 } & $\mathrm{C} / \mathrm{T}(\mathrm{R} 80 \mathrm{Q})$ & $C C$ & $C T$ & & & $C C$ & $C T$ & & \\
\hline & & $131 \pm 24$ & $120 \pm 16$ & & $4.06 \times 10^{-21}$ & $76 \pm 14$ & $75 \pm 12$ & & 0.0471 \\
\hline \multirow[t]{2}{*}{ rs188378669 } & $\mathrm{G} / \mathrm{T}(\mathrm{E} 31 *)$ & $G G$ & $G T$ & & & $G G$ & $G T$ & & \\
\hline & & $131 \pm 24$ & $120 \pm 16$ & & $1.40 \times 10^{-20}$ & $76 \pm 14$ & $75 \pm 12$ & & 0.0912 \\
\hline \multirow[t]{2}{*}{ rs146092501 } & C/T (E1386K) & $C C$ & $C T$ & & & $C C$ & $C T$ & & \\
\hline & & $131 \pm 24$ & $120 \pm 16$ & & $2.45 \times 10^{-20}$ & $76 \pm 14$ & $75 \pm 12$ & & 0.0565 \\
\hline \multirow[t]{2}{*}{ rs146879198 } & G/A (R340*) & $G G$ & $G A$ & & & $G G$ & $G A$ & & \\
\hline & & $131 \pm 24$ & $120 \pm 16$ & & $2.70 \times 10^{-20}$ & $76 \pm 14$ & $75 \pm 12$ & & $\begin{array}{c}0.0982 \\
\text { (Continued) }\end{array}$ \\
\hline
\end{tabular}




\begin{tabular}{|c|c|c|c|c|c|c|c|c|c|}
\hline \multirow{2}{*}{$\frac{\text { SNP }}{\text { rs115287176 }}$} & \multirow[b]{2}{*}{ G/A (R277W) } & \multicolumn{3}{|c|}{ Systolic BP (mmHg) } & \multirow[t]{2}{*}{$P$} & \multicolumn{3}{|c|}{ Diastolic BP (mmHg) } & \multirow[t]{2}{*}{$P$} \\
\hline & & $G G$ & $G A$ & & & $G G$ & $G A$ & & \\
\hline & & $131 \pm 24$ & $120 \pm 16$ & & $5.08 \times 10^{-20}$ & $76 \pm 14$ & $75 \pm 12$ & & 0.0941 \\
\hline \multirow[t]{2}{*}{ rs192210727 } & G/T (R580I) & $G G$ & $G T$ & $T T$ & & $G G$ & $G T$ & $T T$ & \\
\hline & & $131 \pm 24$ & $120 \pm 16$ & $118 \pm 10$ & $8.83 \times 10^{-19}$ & $76 \pm 14$ & $75 \pm 12$ & $70 \pm 11$ & 0.1403 \\
\hline \multirow[t]{2}{*}{ rs199576535 } & G/A (V341I) & $G G$ & $G A$ & & & $G G$ & $G A$ & & \\
\hline & & $131 \pm 24$ & $120 \pm 17$ & & $5.70 \times 10^{-16}$ & $76 \pm 14$ & $75 \pm 14$ & & 0.0243 \\
\hline \multirow[t]{2}{*}{ rs 147284320} & $\mathrm{C} / \mathrm{T}$ (V503I) & $C C$ & $C T$ & & & $C C$ & $C T$ & & \\
\hline & & $128 \pm 21$ & $120 \pm 16$ & & $4.36 \times 10^{-14}$ & $75 \pm 12$ & $75 \pm 12$ & & 0.6346 \\
\hline \multirow[t]{2}{*}{ rs201459911 } & $\begin{array}{c}\mathrm{G} / \mathrm{A} \\
(\mathrm{A} 1115 \mathrm{~V})\end{array}$ & $G G$ & $G A$ & & & $G G$ & $G A$ & & \\
\hline & & $131 \pm 24$ & $143 \pm 28$ & & $1.16 \times 10^{-14}$ & $76 \pm 14$ & $76 \pm 16$ & & 0.4868 \\
\hline \multirow[t]{2}{*}{ rs199976159 } & $\mathrm{G} / \mathrm{A}(\mathrm{G} 222 \mathrm{~S})$ & $G G$ & $G A$ & & & $G G$ & $G A$ & & \\
\hline & & $131 \pm 24$ & $149 \pm 25$ & & $3.98 \times 10^{-9}$ & $76 \pm 14$ & $73 \pm 15$ & & 0.0627 \\
\hline \multirow[t]{2}{*}{ rs 2523638} & $\mathrm{G} / \mathrm{A}$ & $G G$ & $G A$ & $A A$ & & $G G$ & $G A$ & $A A$ & \\
\hline & & $130 \pm 23$ & $131 \pm 24$ & $133 \pm 25$ & $7.24 \times 10^{-8}$ & $76 \pm 13$ & $76 \pm 13$ & $77 \pm 14$ & 0.0002 \\
\hline \multirow[t]{2}{*}{ rs11191454 } & $\mathrm{A} / \mathrm{G}$ & $A A$ & $A G$ & $G G$ & & $A A$ & $A G$ & $G G$ & \\
\hline & & $132 \pm 24$ & $130 \pm 24$ & $128 \pm 22$ & $1.43 \times 10^{-7}$ & $77 \pm 14$ & $76 \pm 13$ & $75 \pm 13$ & 0.0032 \\
\hline \multirow[t]{2}{*}{ rs 12182351} & $\mathrm{~T} / \mathrm{C}$ & $T T$ & $T C$ & $C C$ & & $T T$ & $T C$ & $C C$ & \\
\hline & & $130 \pm 23$ & $132 \pm 25$ & $132 \pm 24$ & $2.43 \times 10^{-9}$ & $76 \pm 13$ & $77 \pm 14$ & $76 \pm 14$ & 0.0010 \\
\hline \multirow[t]{2}{*}{ rs12413409 } & $\mathrm{G} / \mathrm{A}$ & $G G$ & $G A$ & $A A$ & & $G G$ & $G A$ & $A A$ & \\
\hline & & $132 \pm 24$ & $130 \pm 24$ & $128 \pm 22$ & $2.03 \times 10^{-7}$ & $77 \pm 14$ & $76 \pm 14$ & $75 \pm 13$ & 0.0029 \\
\hline \multirow[t]{2}{*}{ rs144936999 } & $\mathrm{G} / \mathrm{C}(\mathrm{A} 332 \mathrm{P})$ & $G G$ & $G C$ & & & $G G$ & $G C$ & & \\
\hline & & $131 \pm 24$ & $154 \pm 32$ & & $4.16 \times 10^{-8}$ & $76 \pm 14$ & $76 \pm 15$ & & 0.9989 \\
\hline \multirow[t]{2}{*}{ rs 404890} & $\mathrm{G} / \mathrm{T}$ & $G G$ & $G T$ & $T T$ & & $G G$ & $G T$ & $T T$ & \\
\hline & & $130 \pm 23$ & $132 \pm 25$ & $132 \pm 24$ & $7.91 \times 10^{-9}$ & $76 \pm 13$ & $77 \pm 14$ & $76 \pm 14$ & 0.0018 \\
\hline \multirow[t]{2}{*}{ rs 130075} & C/T (R102Q) & $C C$ & $C T$ & $T T$ & & $C C$ & $C T$ & $T T$ & \\
\hline & & $132 \pm 24$ & $129 \pm 23$ & $129 \pm 22$ & $8.48 \times 10^{-8}$ & $76 \pm 14$ & $76 \pm 13$ & $75 \pm 14$ & 0.0152 \\
\hline \multirow[t]{2}{*}{ rs11191580 } & $\mathrm{T} / \mathrm{C}$ & $T T$ & $T C$ & $C C$ & & $T T$ & $T C$ & $C C$ & \\
\hline & & $132 \pm 24$ & $130 \pm 24$ & $128 \pm 22$ & $4.44 \times 10^{-7}$ & $77 \pm 14$ & $76 \pm 13$ & $75 \pm 13$ & 0.0027 \\
\hline \multirow[t]{2}{*}{ rs 1058026} & $\mathrm{~T} / \mathrm{G}$ & $T T$ & $T G$ & $G G$ & & $T T$ & $T G$ & $G G$ & \\
\hline & & $132 \pm 24$ & $130 \pm 24$ & $129 \pm 22$ & $6.18 \times 10^{-7}$ & $77 \pm 14$ & $76 \pm 13$ & $76 \pm 13$ & 0.1202 \\
\hline \multirow[t]{2}{*}{ rs11191548 } & $\mathrm{T} / \mathrm{C}$ & $T T$ & $T C$ & $C C$ & & $T T$ & $T C$ & $C C$ & \\
\hline & & $132 \pm 24$ & $130 \pm 24$ & $128 \pm 22$ & $6.26 \times 10^{-7}$ & $77 \pm 14$ & $76 \pm 13$ & $75 \pm 13$ & 0.0033 \\
\hline \multirow[t]{2}{*}{ rs2270191 } & $\mathrm{C} / \mathrm{T}(\mathrm{V} 5 \mathrm{M})$ & $C C$ & $C T$ & $T T$ & & $C C$ & $C T$ & $T T$ & \\
\hline & & $132 \pm 24$ & $129 \pm 23$ & $129 \pm 22$ & $4.19 \times 10^{-7}$ & $76 \pm 14$ & $76 \pm 13$ & $75 \pm 14$ & 0.0490 \\
\hline
\end{tabular}

(Continued) 


\begin{tabular}{|c|c|c|c|c|c|c|c|c|c|}
\hline \multicolumn{2}{|l|}{ SNP } & \multicolumn{3}{|c|}{ Systolic BP (mmHg) } & \multirow[t]{2}{*}{$P$} & \multicolumn{3}{|c|}{ Diastolic BP (mmHg) } & \multirow[t]{2}{*}{$\boldsymbol{P}$} \\
\hline rs117951780 & $\mathrm{C} / \mathrm{T}(\mathrm{S} 453 \mathrm{~N})$ & $C C$ & $C T$ & $T T$ & & $C C$ & $C T$ & $T T$ & \\
\hline & & $132 \pm 24$ & $129 \pm 23$ & $129 \pm 22$ & $5.95 \times 10^{-7}$ & $76 \pm 14$ & $76 \pm 13$ & $75 \pm 14$ & 0.0461 \\
\hline \multirow[t]{2}{*}{ rs2270190 } & $\mathrm{T} / \mathrm{C}$ & $T T$ & $T C$ & $C C$ & & $T T$ & $T C$ & $C C$ & \\
\hline & & $132 \pm 24$ & $129 \pm 23$ & $129 \pm 22$ & $5.73 \times 10^{-7}$ & $76 \pm 14$ & $76 \pm 13$ & $76 \pm 14$ & 0.0360 \\
\hline \multirow[t]{2}{*}{ rs 17115100} & $\mathrm{G} / \mathrm{T}$ & $G G$ & $G T$ & $T T$ & & $G G$ & $G T$ & $T T$ & \\
\hline & & $132 \pm 24$ & $131 \pm 24$ & $128 \pm 21$ & $3.37 \times 10^{-7}$ & $77 \pm 14$ & $76 \pm 14$ & $75 \pm 13$ & 0.0101 \\
\hline \multirow[t]{2}{*}{ rs 72655343} & $\mathrm{C} / \mathrm{A}$ & $C C$ & $C A$ & & & $C C$ & $C A$ & & \\
\hline & & $131 \pm 24$ & $149 \pm 30$ & & $4.24 \times 10^{-7}$ & $76 \pm 14$ & $77 \pm 17$ & & 0.7231 \\
\hline \multirow[t]{2}{*}{ rs 1063646} & $\mathrm{C} / \mathrm{T}(\mathrm{P} 133 \mathrm{~L})$ & $C C$ & $C T$ & $T T$ & & $C C$ & $C T$ & $T T$ & \\
\hline & & $132 \pm 24$ & $129 \pm 23$ & $130 \pm 23$ & $6.24 \times 10^{-7}$ & $76 \pm 14$ & $76 \pm 13$ & $76 \pm 14$ & 0.0473 \\
\hline \multirow[t]{2}{*}{ rs9263739 } & $\mathrm{C} / \mathrm{T}$ & $C C$ & $C T$ & $T T$ & & $C C$ & $C T$ & $T T$ & \\
\hline & & $132 \pm 24$ & $129 \pm 23$ & $130 \pm 23$ & $6.24 \times 10^{-7}$ & $76 \pm 14$ & $76 \pm 13$ & $76 \pm 14$ & 0.0473 \\
\hline \multirow[t]{2}{*}{ rs 1004467} & $\mathrm{~T} / \mathrm{C}$ & $T T$ & $T C$ & $C C$ & & $T T$ & $T C$ & $C C$ & \\
\hline & & $132 \pm 24$ & $131 \pm 24$ & $128 \pm 21$ & $2.28 \times 10^{-6}$ & $77 \pm 14$ & $76 \pm 14$ & $75 \pm 13$ & 0.0208 \\
\hline \multirow[t]{2}{*}{ rs139421991 } & G/A (R320Q) & $G G$ & $G A$ & & & $G G$ & $G A$ & & \\
\hline & & $131 \pm 24$ & $143 \pm 28$ & & $6.74 \times 10^{-7}$ & $76 \pm 14$ & $79 \pm 18$ & & 0.0280 \\
\hline \multirow[t]{2}{*}{ rs7757012 } & $\mathrm{T} / \mathrm{C}$ & $T T$ & $T C$ & $C C$ & & $T T$ & $T C$ & $C C$ & \\
\hline & & $132 \pm 24$ & $129 \pm 23$ & $130 \pm 23$ & $1.02 \times 10^{-6}$ & $76 \pm 14$ & $76 \pm 13$ & $76 \pm 13$ & 0.0526 \\
\hline \multirow[t]{2}{*}{ rs142979264 } & $\mathrm{C} / \mathrm{T}$ & $C C$ & $C T$ & $T T$ & & $C C$ & $C T$ & $T T$ & \\
\hline & & $132 \pm 24$ & $129 \pm 23$ & $128 \pm 21$ & $3.71 \times 10^{-6}$ & $76 \pm 14$ & $76 \pm 13$ & $76 \pm 13$ & 0.0756 \\
\hline \multirow[t]{2}{*}{ rs10849915 } & $\mathrm{T} / \mathrm{C}$ & $T T$ & $T C$ & $C C$ & & $T T$ & $T C$ & $C C$ & \\
\hline & & $132 \pm 24$ & $130 \pm 24$ & $129 \pm 23$ & $7.27 \times 10^{-6}$ & $77 \pm 14$ & $76 \pm 13$ & $75 \pm 13$ & $7.60 \times 10^{-5}$ \\
\hline \multicolumn{10}{|c|}{ Associated with diastolic BP } \\
\hline \multirow[t]{2}{*}{ rs7969300 } & $\mathrm{T} / \mathrm{C}(\mathrm{N} 248 \mathrm{~S})$ & $T T$ & $T C$ & $C C$ & & $T T$ & $T C$ & $C C$ & \\
\hline & & $130 \pm 24$ & $131 \pm 24$ & $133 \pm 25$ & 0.0039 & $76 \pm 14$ & $76 \pm 14$ & $78 \pm 14$ & $4.59 \times 10^{-8}$ \\
\hline \multirow[t]{2}{*}{ rs12231744 } & C/T (R876K) & $C C$ & $C T$ & $T T$ & & $C C$ & $C T$ & $T T$ & \\
\hline & & $131 \pm 24$ & $131 \pm 24$ & $133 \pm 25$ & 0.0145 & $76 \pm 14$ & $76 \pm 14$ & $78 \pm 14$ & $1.44 \times 10^{-7}$ \\
\hline \multicolumn{10}{|c|}{ Associated with hypertension } \\
\hline \multirow[t]{2}{*}{ rs150854849 } & C/T (R179Q) & $C C$ & $C T$ & & & $C C$ & $C T$ & & \\
\hline & & $131 \pm 25$ & $133 \pm 23$ & & 0.5574 & $76 \pm 14$ & $76 \pm 10$ & & 0.7538 \\
\hline \multirow[t]{2}{*}{ rs202069030 } & G/C (R51S) & $G G$ & $G C$ & & & $G G$ & $G C$ & & \\
\hline & & $131 \pm 24$ & $131 \pm 24$ & & 0.9913 & $76 \pm 14$ & $75 \pm 10$ & & 0.6866 \\
\hline \multirow[t]{2}{*}{ rs 139012426} & $\mathrm{G} / \mathrm{C}(\mathrm{S} 1242 \mathrm{~T})$ & $G G$ & $G C$ & & & $G G$ & $G C$ & & \\
\hline & & $131 \pm 24$ & $138 \pm 26$ & & 0.1728 & $76 \pm 14$ & $77 \pm 9$ & & 0.8428 \\
\hline \multirow[t]{2}{*}{ rs76974938 } & $\mathrm{C} / \mathrm{T}(\mathrm{D} 67 \mathrm{~N})$ & $C C$ & $C T$ & & & $C C$ & $C T$ & & \\
\hline & & $131 \pm 25$ & $132 \pm 19$ & & 0.1772 & $76 \pm 14$ & $75 \pm 11$ & & 0.0291 \\
\hline
\end{tabular}

Data are compared among genotypes by one-way analysis of variance. Based on Bonferroni's correction, $P$ values of $<0.0005(0.05 / 100)$ were considered statistically significant and are shown in bold. 


\section{DISCUSSION}

We have now shown that rs12229654 at $12 \mathrm{q} 24.1$ was significantly associated with both systolic and diastolic BP as well as with hypertension and that rs671 of $A L D H 2$, rs11066015 of $A C A D 10$, rs2074356 and rs 11066280 of HECTD4, and rs3782886 of BRAP were significantly associated with both systolic and diastolic BP. Among these loci, ALDH2 and HECTD4 were identified as susceptibility loci for BP in a previous GWAS [10]. The remaining SNPs at three loci (12q24.1, ACAD10, and $B R A P)$ are newly identified as genetic determinants of systolic and diastolic BP in Japanese. We also uncovered an additional 38 and two SNPs that were associated with systolic or diastolic BP, respectively, as well as four SNPs associated with hypertension.

The six SNPs (rs12229654, rs671, rs11066015, rs2074356, rs3782886, rs11066280) significantly associated with BP are clustered at 12q24.12-q24.13 and are in linkage disequilibrium with each other. The SNP rs 12229654 at $12 \mathrm{q} 24.1$ was previously shown to be associated with body mass index [15] and metabolic syndrome [16], with obesity and metabolic syndrome both being risk factors for elevated BP. The aldehyde dehydrogenase 2 family gene $(A L D H 2)$ is located at 12q24.12 (NCBI Gene, https://www.ncbi.nlm.nih.gov/ gene) and is expressed in various organs and tissues, especially the liver (The Human Protein Atlas, http:// www.proteinatlas.org). ALDH2 catalyze the second step of the major oxidative pathway of alcohol metabolism (NCBI Gene). ALDH2 was previously shown to be associated with $\mathrm{BP}$ and hypertension $[8,10]$ The acylCoA dehydrogenase family member 10 gene (ACAD10) is located at 12q24.12 (NCBI Gene), is ubiquitously expressed (The Human Protein Atlas), and activates the $\beta$-oxidation of fatty acids in mitochondria (NCBI Gene). $A C A D 10$ has not previously been associated with BP or hypertension, although rs11066015 of this gene was shown to be related to coronary artery disease [17]. The HECT domain E3 ubiquitin protein ligase 4 gene (HECTD4) is located at 12q24.13 (NCBI Gene) and is broadly expressed (The Human Protein Atlas). E3 ligases accept ubiquitin from an E2 ubiquitinconjugating enzyme in the form of a thioester and then directly transfer the ubiquitin moiety to target substrates [Universal Protein Resource, http://www.uniprot. org]. HECTD4 was previously found to be associated with systolic and diastolic BP $[10,18]$. The BRCA1 associated protein gene $(B R A P)$ is located at $12 \mathrm{q} 24.12$ (NCBI Gene) and is ubiquitously expressed, with its expression level being especially high in the testis (The Human Protein Atlas). BRAP has not previously been associated with BP or hypertension, although it was found to be a susceptibility locus for myocardial infarction [19].
In recent large-scale GWASs, the MAFs of BPassociated SNPs were 5\% to 50\% (mean, 29.8\%) and their effect sizes for systolic or diastolic BP were 0.07 to 1.13 $\mathrm{mmHg}$ (mean, $0.46 \mathrm{mmHg}$ ) and 0.06 to $0.6 \mathrm{mmHg}$ (mean, $0.28 \mathrm{mmHg}$ ), respectively [20]. In our study, the MAFs of the six SNPs (rs12229654, rs671, rs11066015, rs2074356, rs3782886, rs11066280) associated with both systolic and diastolic BP were $22.5 \%$ to $29.3 \%$ and the differences in systolic or diastolic BP among genotypes were 3 to 4 $\mathrm{mmHg}$ and 2 to $3 \mathrm{mmHg}$, respectively. These SNPs were thus common variants with moderate effect sizes. We also detected an additional 38 and two SNPs associated with systolic or diastolic BP, respectively. Nineteen of these 38 SNPs associated with systolic BP had a MAF of $0.1 \%$ to $2.0 \%$ and the corresponding differences in systolic BP among (or between) genotypes were 11 to $23 \mathrm{mmHg}$; these SNPs were thus low-frequency or rare variants with large effect sizes. The remaining 19 SNPs were common variants (MAF, $11.8 \%$ to $43.1 \%$ ) with differences in systolic BP among (or between) genotypes being 2 to 4 $\mathrm{mmHg}$. The additional two SNPs associated with diastolic BP were common variants (MAF, $35.1 \%$ and $38.8 \%$ ), with the differences in diastolic BP among genotypes being 2 $\mathrm{mmHg}$.

There were several limitations to the present study: (1) Given that the results were not replicated, they will require validation in other independent populations or in other ethnic groups. (2) Given that subjects were recruited by different methods, hypertension may be a more reliable phenotype compared with $\mathrm{BP}$ in the present study. Therefore the relation of SNPs to systolic and diastolic BP should be interpreted carefully. (3) Given that the prevalence of hypertension was higher in the subjects recruited from the hospitals than in those from the general population, selection bias could not be excluded. (4) It is possible that the SNPs identified in the present study are in linkage disequilibrium with other polymorphisms in nearby genes that are actually responsible for the regulation of BP or the development of hypertension. (5) Four SNPs identified as susceptibility loci for hypertension were not associated with systolic or diastolic BP, possibly as a result of the effect of antihypertensive treatment. (6) The functional relevance of the identified SNPs to the pathogenesis of hypertension remains to be determined.

In conclusion, we have identified six SNPs (rs12229654 at 12q24.1, rs671 of $A L D H 2$, rs11066015 of $A C A D 10$, rs2074356 and rs11066280 of HECTD4, and rs3782886 of $B R A P$ ) as genetic determinants of BP. Among these loci, $A L D H 2$ and HECTD4 were previously associated with BP [10]. The remaining SNPs at three loci (12q24.1, $A C A D 10$, and $B R A P$ ) are newly identified as determinants of BP in Japanese. Determination of genotypes for these SNPs may prove informative for assessment of the genetic risk for hypertension in Japanese. 


\section{MATERIALS AND METHODS}

\section{Study subjects}

A total of 14,678 subjects (8215 patients with hypertension and 6463 control individuals) was examined. The subjects were recruited from individuals as described previously [21].

The subjects with hypertension either had a systolic $\mathrm{BP}$ of $\geq 140 \mathrm{mmHg}$ or diastolic BP of $\geq 90 \mathrm{mmHg}$ (or both) or had taken antihypertensive medication. Individuals with severe valvular heart disease, congenital malformations of the heart or vessels, renal or endocrinologic diseases that cause secondary hypertension, or drug-induced hypertension were excluded from the study. The control individuals had a systolic BP of $<140 \mathrm{mmHg}$ and diastolic BP of $<90 \mathrm{mmHg}$ as well as no history of hypertension or of taking antihypertensive medication. Autopsy cases were excluded from controls. BP was measured at least twice with subjects having first rested in the sitting position for $>5 \mathrm{~min}$; the measurements were taken by a skilled physician or nurse according to the guidelines of the American Heart Association [22].

\section{EWASs for systolic or diastolic BP and for hypertension}

Methods for collection and extraction of genomic DNA samples were described previously [21]. The EWASs were performed with the use of a HumanExome-12 v1.1 or v1.2 DNA Analysis BeadChip or Infinium Exome-24 v1.0 BeadChip (Illumina, San Diego, CA, USA). Exome array contains $\sim 244,000$ SNPs including common, low frequency, and rare variants located at whole exons. The GWAS makes use of high-throughput genotyping technologies that include up to 4.5 million markers for SNPs and copy number variations to examine their relation to clinical conditions or traits. The EWAS is a focus genotyping method that differs from the GWAS [23]. Detailed information of the exome arrays and methods of quality control were described previously [21]. A total of 41,843 SNPs passed quality control and was subjected to analysis.

\section{Statistical analysis}

For analysis of characteristics of the study subjects, quantitative data were compared between patients with hypertension and controls with the unpaired Student's $t$ test. Categorical data were compared between the two groups with Fisher's exact test. Allele frequencies were estimated by the gene counting method, and Fisher's exact test was applied to identify departure from HardyWeinberg equilibrium. The relation of genotype of each SNP to systolic or diastolic BP in the EWAS was analyzed with a linear regression model. Allele frequencies of SNPs were compared between subjects with hypertension and controls in the EWAS with Fisher's exact test. To compensate for multiple comparisons of genotypes with hypertension, we applied Bonferroni's correction for statistical significance of association. Given that 41,843 SNPs were analyzed, the significance level was set at $P<1.19 \times 10^{-6}(0.05 / 41,843)$ for each EWAS. Quantile-quantile plots for $P$ values of genotypes or allele frequencies in the EWASs for systolic or diastolic BP or for hypertension are shown in Supplementary Figure 2. The inflation factor $(\lambda)$ was 0.95 for systolic BP, 1.05 for diastolic BP, and 1.11 for hypertension. Multivariable logistic regression analysis was performed with hypertension as a dependent variable and independent variables including age, sex (0, woman; 1, man), and genotype of each SNP. A detailed method of analysis was described previously [21]. The relation of genotypes of isolated SNPs to systolic or diastolic BP was examined with one-way ANOVA. Bonferron's correction was also applied to other analyses as indicated. Statistical tests were performed with JMP Genomics version 6.0 software (SAS Institute, Cary, NC, USA).

\section{Author contributions}

Y. Yamada contributed to conception and design of the study; to acquisition, analysis, and interpretation of the data; and to drafting of the manuscript. J. Sakuma, I. Takeuchi, and Y. Yasukochi contributed to analysis and interpretation of the data as well as to revision of the manuscript. K. Kato, M. Oguri, T. Fujimaki, H. Horibe, M. Muramatsu, M. Sawabe, Y. Fujiwara, Y. Taniguchi, S. Obuchi, H. Kawai, S. Shinkai, S. Mori, and T. Arai each contributed to acquisition of the data and to revision of the manuscript. M. Tanaka contributed to acquisition, analysis, and interpretation of the data as well as to revision of the manuscript. All authors approved submission of the final version of the article for publication.

\section{CONFLICTS OF INTEREST}

The authors declare no conflicts of interest.

\section{FUNDING}

This work was supported by CREST (grant number, JPMJCR1302), Japan Science and Technology Agency (to Y. Yamada, J. Sakuma, and I. Takeuchi) and by Japan Society for the Promotion of Science KAKENHI grants JP15H04772 (to Y. Yamada), JP25242062 (to M. Tanaka), and JP16H01872 (to M. Tanaka).

\section{REFERENCES}

1. Rapsomaniki E, Timmis A, George J, Pujades-Rodriguez M, Shah AD, Denaxas S, White IR, Caulfield MJ, Deanfield 
JE, Smeeth L, Williams B, Hingorani A, Hemingway H. Blood pressure and incidence of twelve cardiovascular diseases: lifetime risks, healthy life-years lost, and agespecific associations in 1.25 million people. Lancet. 2014; 383:1899-1911.

2. Agarwal A, Williams GH, Fisher ND. Genetics of human hypertension. Trends Endocrinol Metab. 2005; 16:127-133.

3. Wellcome Trust Case Control Consortium. Genome-wide association study of 14,000 cases of seven common diseases and 3,000 shared controls. Nature. 2007; 447:661-678.

4. Levy D, Ehret GB, Rice K, Verwoert GC, Launer LJ, Dehghan A, Glazer NL, Morrison AC, Johnson AD, Aspelund T, Aulchenko Y, Lumley T, Köttgen A, et al. Genome-wide association study of blood pressure and hypertension. Nat Genet. 2009; 41:677-687.

5. Newton-Cheh C, Johnson T, Gateva V, Tobin MD, Bochud M, Coin L, Najjar SS, Zhao JH, Heath SC, Eyheramendy S, Papadakis K, Voight BF, Scott LJ, et al. Genome-wide association study identifies eight loci associated with blood pressure. Nat Genet. 2009; 41:666-676.

6. International Consortium for Blood Pressure Genome-Wide Association Studies, Ehret GB, Munroe PB, Rice KM, Bochud M, Johnson AD, Chasman DI, Smith AV, Tobin MD, Verwoert GC, Hwang SJ, Pihur V, Vollenweider P, et al. Genetic variants in novel pathways influence blood pressure and cardiovascular disease risk. Nature. 2011; 478:103-109.

7. Adeyemo A, Gerry N, Chen G, Herbert A, Doumatey A, Huang H, Zhou J, Lashley K, Chen Y, Christman M, Rotimi C. A genome-wide association study of hypertension and blood pressure in African Americans. PLoS Genet. 2009; 5:e1000564.

8. Lu X, Wang L, Lin X, Huang J, Charles Gu C, He M, Shen H, He J, Zhu J, Li H, Hixson JE, Wu T, Dai J, et al. Genome-wide association study in Chinese identifies novel loci for blood pressure and hypertension. Hum Mol Genet. 2015; 24:865-874.

9. Kato N, Miyata T, Tabara Y, Katsuya T, Yanai K, Hanada H, Kamide K, Nakura J, Kohara K, Takeuchi F, Mano H, Yasunami M, Kimura A, et al. High-density association study and nomination of susceptibility genes for hypertension in the Japanese National Project. Hum Mol Genet. 2008; 17:617-627.

10. Kato N, Loh M, Takeuchi F, Verweij N, Wang X, Zhang W, Kelly TN, Saleheen D, Lehne B, Mateo Leach I, Drong AW, Abbott J, Wahl S, et al. Trans-ancestry genome-wide association study identifies 12 genetic loci influencing blood pressure and implicates a role for DNA methylation. Nat Genet. 2015; 47:1282-1293.

11. Manolio TA, Collins FS, Cox NJ, Goldstein DB, Hindorff LA, Hunter DJ, McCarthy MI, Ramos EM, Cardon LR, Chakravarti A, Cho JH, Guttmacher AE, Kong A, et al. Finding the missing heritability of complex diseases. Nature. 2009; 461:747-753.
12. Simino J, Shi G, Bis JC, Chasman DI, Ehret GB, Gu X, Guo X, Hwang SJ, Sijbrands E, Smith AV, Verwoert GC, Bragg-Gresham JL, Cadby G, et al. Gene-age interactions in blood pressure regulation: a large-scale investigation with the CHARGE, Global BPgen, and ICBP Consortia. Am J Hum Genet. 2014; 95:24-38.

13. Wain LV, Verwoert GC, O'Reilly PF, Shi G, Johnson T, Johnson AD, Bochud M, Rice KM, Henneman P, Smith AV, Ehret GB, Amin N, Larson MG, et al. Genome-wide association study identifies six new loci influencing pulse pressure and mean arterial pressure. Nat Genet. 2011; 43:1005-1011.

14. Kato N, Takeuchi F, Tabara Y, Kelly TN, Go MJ, Sim X, Tay WT, Chen CH, Zhang Y, Yamamoto K, Katsuya T, Yokota M, Kim YJ, et al. Meta-analysis of genome-wide association studies identifies common variants associated with blood pressure variation in east Asians. Nat Genet. 2011; 43:531-538.

15. Wen W, Zheng W, Okada Y, Takeuchi F, Tabara Y, Hwang JY, Dorajoo R, Li H, Tsai FJ, Yang X, He J, Wu Y, He M, et al. Meta-analysis of genome-wide association studies in East Asian-ancestry populations identifies four new loci for body mass index. Hum Mol Genet. 2014; 23:5492-5504.

16. Shim U, Kim HN, Sung YA, Kim HL. Pathway analysis of metabolic syndrome using a genome-wide association study of Korea Associated Resource (KARE) cohorts. Genomics Inform. 2014; 12:195-202.

17. Lee JY, Lee BS, Shin DJ, Woo Park K, Shin YA, Joong Kim K, Heo L, Young Lee J, Kyoung Kim Y, Jin Kim Y, Bum Hong C, Lee SH, Yoon D, et al. A genome-wide association study of a coronary artery disease risk variant. J Hum Genet. 2013; 58:120-126.

18. Zhang H, Mo XB, Xu T, Bu XQ, Lei SF, Zhang YH. Novel genes affecting blood pressure detected via gene-based association analysis. G3 (Bethesda). 2015; 5:1035-1042.

19. Hirokawa M, Morita H, Tajima T, Takahashi A, Ashikawa K, Miya F, Shigemizu D, Ozaki K, Sakata Y, Nakatani D, Suna S, Imai Y, Tanaka T, et al. A genome-wide association study identifies PLCL2 and AP3D1-DOT1L-SF3A2 as new susceptibility loci for myocardial infarction in Japanese. Eur J Hum Genet. 2015; 23:374-380.

20. Ehret GB, Ferreira T, Chasman DI, Jackson AU, Schmidt EM, Johnson T, Thorleifsson G, Luan J, Donnelly LA, Kanoni S, Petersen AK, Pihur V, Strawbridge RJ, et al. The genetics of blood pressure regulation and its target organs from association studies in 342,415 individuals. Nat Genet. 2016; 48:1171-1184.

21. Yamada Y, Sakuma J, Takeuchi I, Yasukochi Y, Kato K, Oguri M, Fujimaki T, Horibe H, Muramatsu M, Sawabe M, Fujiwara Y, Taniguchi Y, Obuchi S, et al. Identification of STXBP2 as a novel susceptibility locus for myocardial infarction in Japanese individuals by an exome-wide association study. Oncotarget. 2017; 8:33527-33535. doi: 10.18632/oncotarget.16536. 
22. Perloff D, Grim C, Flack J, Frohlich ED, Hill M, McDonald M, Morgenstern BZ. Human blood pressure determination by sphygmomanometry. Circulation. 1993; 88:2460-2470.

23. Yamada Y, Sakuma J, Takeuchi I, Yasukochi Y, Kato K, Oguri M, Fujimaki T, Horibe H, Muramatsu M, Sawabe M,
Fujiwara Y, Taniguchi Y, Obuchi S, et al. Identification of C21orf59 and ATG2A as novel determinants of renal function-related traits in Japanese by exomewide association studies. Oncotarget. 2017 Mar 30. doi: 10.18632/oncotarget.16696. [Epub ahead of print]. 